Convenient diastereoselective synthesis of annulated 3-substituted- $\left(5 S^{*}, 6 S^{*}, Z\right)$ -

\author{
Hassan, Alaa A.
}

2019-11

Hassan , A A , Mohamed , N K, Aly , A A , Tawfeek , H N , Hopf , H , Bräse , S \& Nieger , M 2019 , ' Convenient diastereoselective synthesis of annulated

3-substituted-(5S*,6S* Z)-2-(2-(2,4-dinitrophenyl)hydrazono)-5,6-diphenyl-1,3-thiazinan-4-ones

' , Molecular Diversity , vol. 23 , no. 4 , pp. 821-828 . https://doi.org/10.1007/s11030-018-09912-5

http://hdl.handle.net/10138/314834

https://doi.org/10.1007/s11030-018-09912-5

acceptedVersion

Downloaded from Helda, University of Helsinki institutional repository.

This is an electronic reprint of the original article.

This reprint may differ from the original in pagination and typographic detail.

Please cite the original version. 


\section{Convenient Diastereoselective Synthesis of Annulated 3-}

2 substituted-(5S*,6 $\left.S^{*}, Z\right)-2-(2-(2,4-d i n i t r o p h e n y l) h y d r a z o n o)-5,6-$

3 diphenyl-1,3-thiazinan-4-ones

4

5 Alaa A. Hassan, ${ }^{1 *}$ Nasr K. Mohamed, ${ }^{1}$ Ashraf A. Aly, ${ }^{1}$ Hendawy N. Tawfeek, ${ }^{1}$

6 Henning Hopf, ${ }^{2}$ Stefan Bräse, ${ }^{3}$ Martin Nieger ${ }^{4}$

7

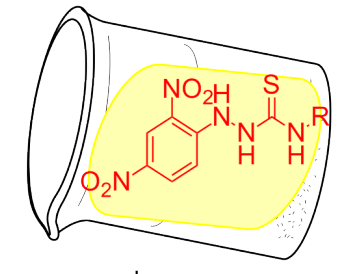

$\mathrm{EtOH}$, reflux

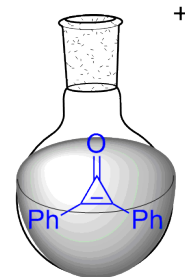

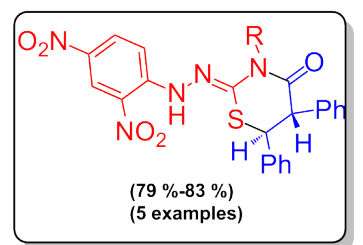

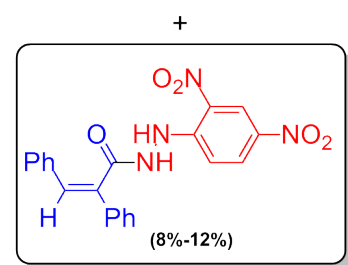

Keywords:

1,3-Thiazinan-4-ones. Substituted 2,3-diphenylacrylohydrazide. (2,4Dinitrophenyl)-4-substituted thiosemicarbazides. 2,3-Diphenylcyclopropenone. Annulated compounds.
Racemic 2-(2,4-dinitrophenyl)hydrazono)-5,6-diphenyl-1,3-thiazinan-4ones and $(Z)-N^{\prime}$-(2,4-dinitrophenyl)-2,3-diphenylacrylohydrazide were formed during the diastereoselective reaction between 4 -substituted 1(2,4-dinitrophenyl)thiosemicarbazides and 2,3-diphenylcycloprop-2enone under refluxing ethanol. The structures of the synthesized compounds were confirmed by single crystal X-ray analyses.

${ }^{1}$ Chemistry Department, Faculty of Science, Minia University, El-Minia 61519, Egypt .

${ }^{2}$ Institut für Organische Chemie, Technische Universität Braunschweig, D-38092 Braunschweig,

${ }^{3}$ Institute of Organic Chemistry, Karlsruhe Institute of Technology, Fritz-Haber-Weg 6, 76131 Karlsruhe, Germany. Helsinki, Finland. 


\section{Introduction}

Thiazinanones, despite being rarely reported, are very interesting compounds due to their important role in medicinal chemistry [1-3]. Substituted thiazinanones exhibited antitumor [4], antifungal activity [5] and antimalarial activity which evaluated by Kumawat et al. [6], as well as anti-oxidant activity [7]. Reactions containing an amine, carbonyl compounds and a mercapto acid in one-pot three-component condensation or a two-step process afforded thiazinanone derivatives [5]. 3-Alkyl-2-aryl-1,3-thiazinan-4ones containing a methylsulfonyl pharmacophore were synthesized and their cyclooxygenase-2-[COX-2] inhibitory activity have been evaluated [8]. 3-Pyridin-2-ylmethyl-1,3-thiazinan-4-ones were synthesized and their anti-oxidant activities were evaluated [7].

On the other hand, the behavior of 2,3-diphenylcyclopropenone 1 towards compounds containing $\mathrm{C}=\mathrm{N}$ moieties with the formation of aza-cyclopentanones (pyrrolinones) have been reported [9-12].

Due to aza-enamine reactivity shown by the reaction of alkenylidenehydrazinecarbothioamides 2 with cyclopropenone $\mathbf{1}$ and the availability of azomethine carbon as well as sulfur atom as nucleophilic sites; 3,5-disubstituted 1,3,4-thiadiazolyl-2,3-diphenylpropenones 3 were formed [13] (Scheme 1).

The reaction of 1 with various aldehyde 4-phenylthiosemicarbazones in acetic acid provided, pyrrolo[2,1-b]oxadiazoles [14]. Also, thione derivatives such as 2,4-disubstituted thiosemicarbazides $(4, \mathrm{R}=$ $\mathrm{C}_{6} \mathrm{H}_{5} ; \mathrm{C}_{6} \mathrm{H}_{5} \mathrm{CH}_{2}$ ) reacted with $\mathbf{1}$ via a nucleophilic attack of terminal- $\mathrm{NH}_{2}$ of $\mathbf{4}$ on the carbonyl group of $\mathbf{1}$, afforded pyridazines 5 (Scheme 1) [15].

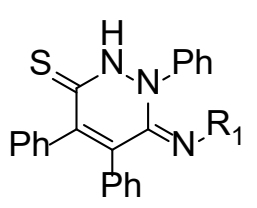

5

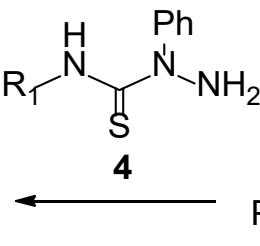

1

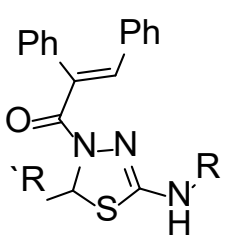

3

Scheme 1: Previously reported interaction of 2,3-diphenylcyclopropenone 1 with alkenylidene hydrazinecarbothioamides $\mathbf{2}$ and 2,4-disubstituted thiosemicarbazides 4.

Optically active sulfur compounds play an important role in the biochemistry of many living organisms and are found in many synthetic drugs and bioactive natural products $[16,17]$. Recently, the [3+3] cyclization of amides with cyclopropenethiones afforded the formation of $6 H-1,3$-oxazin-6-ones and $6 H-1,3-$ thiazin-6-ones [18]. 


\section{Results and discussion}

Herein, we report the reaction of 1-(2,4-dinitrophenyl)-4-substituted thiosemicarbazides 6a-e with 2,3-diphenylcyclopropenone 1 in absolute ethanol under reflux, (5S*,6 $\left.S^{*}, Z\right)-2-(2-(2,4$-dinitrophenyl)hydrazono)-3-substituted-5,6-diphenyl-1,3-thiazinan-4-ones 7a-e were precipitated as a major product (79 $\%-83 \%)$. The filtrate was subjected under chromatographic plates to give only one product namely, $(Z)-N^{\prime}-$ (2,4-dinitrophenyl)-2,3-diphenylacrylohydrazide 8 as a minor product (8\%-12\%) (Scheme 2).

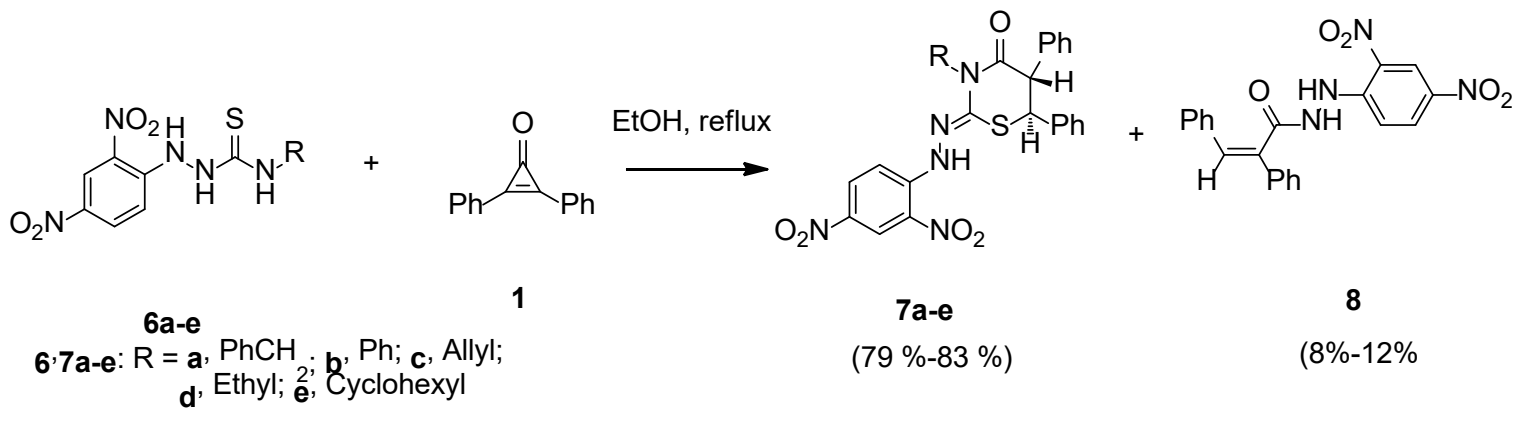

\begin{tabular}{|c|c|c|c|}
\hline Product & $\mathrm{R}$ & Yield (\%) & $\begin{array}{c}\text { Yield of } 8 \\
(\%)\end{array}$ \\
\hline $\mathbf{7 a}$ & $\mathrm{PhCH}_{2}$ & 81 & 9 \\
\hline $\mathbf{7 b}$ & $\mathrm{Ph}$ & 83 & 8 \\
\hline $\mathbf{7 c}$ & Allyl & 80 & 10 \\
\hline $\mathbf{7 d}$ & Ethyl & 79 & 12 \\
\hline $\mathbf{7 e}$ & Cyclohexyl & 80 & 8 \\
\hline
\end{tabular}

Scheme 2: Preparation of 2-hydrazothiazinan-4-one derivatives 7a-e and (Z)- $N^{\prime}-(2,4-$ dinitrophenyl)-2,3-diphenylacrylohydrazide $\mathbf{8}$.

From the structural investigation, IR spectra of 7a-e showed the stretching frequency range between $3265-3229 \mathrm{~cm}^{-1}$ due to NH-stretching, $2935-2922 \mathrm{~cm}^{-1}$ for ali-CH, $1685-1675 \mathrm{~cm}^{-1}$ for $\mathrm{C}=\mathrm{O}, 1616-1612 \mathrm{~cm}^{-1}$ for $\mathrm{C}=\mathrm{N}$ and $1530-1524,1344-1335 \mathrm{~cm}^{-1}$ due to nitro groups.

The ${ }^{1} \mathrm{H}$ NMR spectrum of $7 \mathbf{a}$ (in $\mathrm{CDCl}_{3}$ ) as an example showed a broad singlet at $\delta=11.1 \mathrm{ppm}$ due to $\mathrm{NH}$-group, which was confirmed further by $\mathrm{D}_{2} \mathrm{O}$ exchange experiment. A doublet of doublet as AXsystem signals at 4.57-4.56 and 5.04-5.03 with coupling constant $J=4.0 \mathrm{~Hz}$ because of $\mathrm{CH}-6$ and $\mathrm{CH}-5$ of thiazinanones 7a. The ${ }^{1} \mathrm{H}$ NMR spectra of 7a-e showed the absence of any signals due to $\mathrm{H}-N^{2}$ or $\mathrm{H}-N^{4}$ groups of 6a-e but compound 7a showed a doublet of doublet signals at 5.40-5.43 and 5.64-5.61 with coupling constant $15.0 \mathrm{~Hz}$ for diastereotopic benzyl- $\mathrm{CH}_{2}$ group.

The ${ }^{13} \mathrm{C}$ NMR spectrum of $7 \mathbf{a}$ showed signals at $\delta=47.15$ and $47.90 \mathrm{ppm}$ which were assigned to thiazinanone-CH6,5. Another signals at $56.36 \mathrm{ppm}$ are assigned to $\mathrm{CH}_{2} \mathrm{Ph}, 168.85 \mathrm{ppm}(\mathrm{C}=\mathrm{O}), 146.67 \mathrm{ppm}$ 
$1 \quad(\mathrm{C}=\mathrm{N})$ and $144.61 \mathrm{ppm}\left(\left(\mathrm{NO}_{2}\right)_{2}-\mathrm{Ar}-\mathrm{C}-\mathrm{NH}\right)$. The similarities of ${ }^{1} \mathrm{H}$ NMR spectra (see experimental part)

2 reveal that the five compounds 7 a-e belong to the same gross structure type namely 3 -substituted 2-(2-(2,4-

3 dinitrophenyl)hydrazono)-5,6-diphenyl-1,3-thiazinan-4-ones. The elemental analyses and mass spectrometry

4 of 7a-e clearly showed that the products were formed during the addition of one molecule of 1 to one

5 molecule of 6a-e without any elimination.

6

The X-ray crystallographic structure of compound 7a further supported its relative configurations

7 as (rac-5 $\left.S^{*}, 6 S^{*}, Z\right)-3-$ benzyl-2-(2-(2,4-dinitrophenyl)hydrazono)-5,6-diphenyl-1,3-thiazinan-4-one. The molecular of 7a (Figure 1 and Tables 1-7, in the crystallographic data) revealed furthermore the formation of 3-benzyl-2-(2-(2,4-dinitrophenyl)hydrazono)-5,6-diphenyl-1,3-thiazinan-4-one in the cissiod (Z) structure.

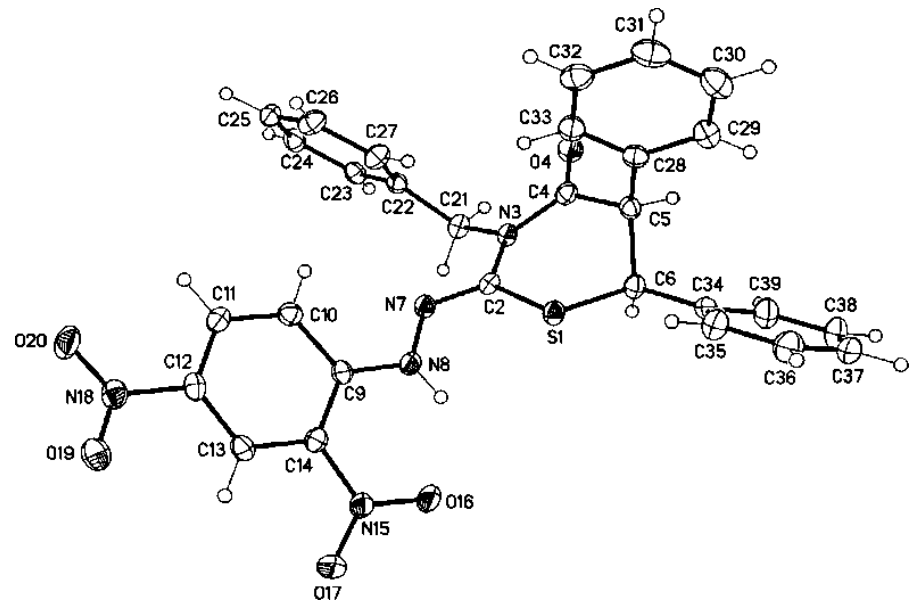

Figure 1: Molecular structure of compound 7a (displacement parameters are drawn at $50 \%$ probability level). The crystallographic numbering does not reflect the IUPAC numbering.

The mechanism for the formation of products $\mathbf{7 a - e}$ is presented in scheme 3 . The sulfur atom attacks the conjugate double bond of $\mathbf{1}$ forming the intermediate $\mathbf{9}$. Intramolecular nucleophilic attack of $N^{4}$ $\mathrm{H}$ on $\mathrm{C}=\mathrm{O}$ afforded the intermediate 11 which rearranged to give 7a-e (Scheme 3). On the other hand, $N^{4}-\mathrm{H}$ attacks the carbonyl group of $\mathbf{1}$ with the formation of $\mathbf{7 a - e}$ via intermediate $\mathbf{1 2}$ (Scheme 3 ).

The ring opening of cyclopropenones has been reported earlier by Gomaa [19] during the reaction of $N^{1}, N^{2}$-diarylformamidines with diphenylcyclopropenone to give 3-aryl-( $N$-4-arylformamidoyl)amino-2,3diphenylpropionic acids.

Recently, Wu et al. reported the ring-opening acylation of cyclopropenones with organo boronic acids afforded $\alpha, \beta$-diaryl unsaturated ketones [20]. 

product ( $8-12 \%$ ) from the reaction of 1 with $\mathbf{6 a - e}$. $\mathrm{cm}^{-1}$ corresponding to carbonyl group and bands at $1528,1344 \mathrm{~cm}^{-1}$ attributed to nitro groups. The ${ }^{1} \mathrm{H}$ NMR spectrum of $\mathbf{8}$ showed multiplet signals at 6.42 due to trisubstituted acrylohydrazide- $\mathrm{CH}, 9.43(\mathrm{NH})$, in addition to the aromatic protons. In the ${ }^{13} \mathrm{C}$ NMR spectrum of $\mathbf{8}$, the signal at $\delta=165.18$ was assigned to amide-CO, 145.10 due to $\left(\left(\mathrm{NO}_{2}\right)_{2}-\mathrm{Ar}-\mathrm{C}-\mathrm{NH}, 133.68\right.$ and 139.75 was attributed to acrylohydrazide $\mathrm{C} 2$ and C3.

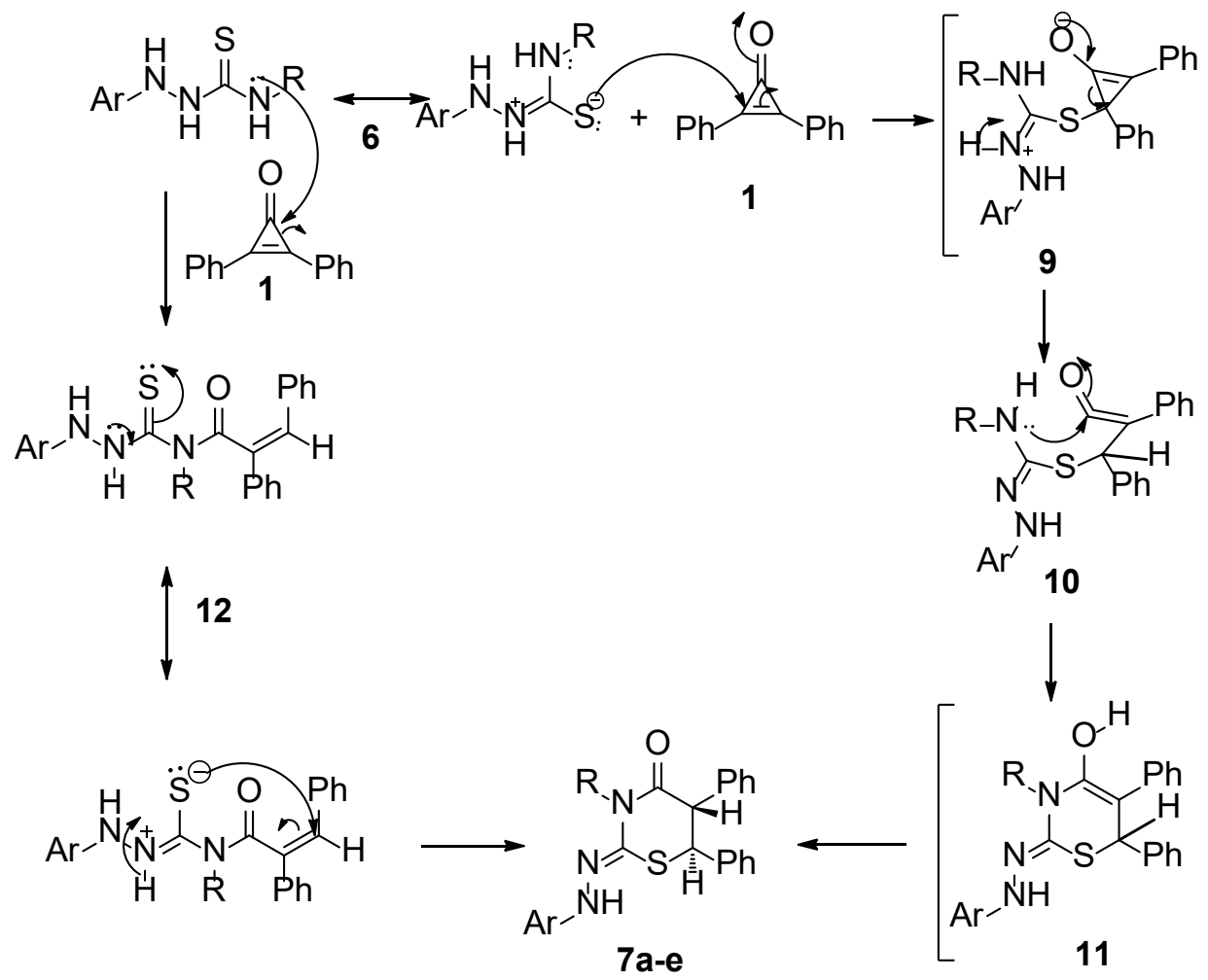

Scheme 3: Mechanism for the formation of 2-hydrazothiazinan-4-one derivatives 7a-e.

The structure of (Z)-N'-(2,4-dinitrophenyl)-2,3-diphenylacrylohydrazide $\mathbf{8}$ was determined by X-ray analysis (Figure 2, Tables 8-15 in the supplementary data). The X-ray structure confirms the trans (E) geometry of the two phenyl groups with respect to the $\mathrm{C} 2-\mathrm{C} 3$ double bond (note that the crystallographic numbering does not correspond to the systematic IUPAC numbering rules).

The hydrazide $\mathbf{8}$ was formed via the nucleophilic addition of $N^{2}-\mathrm{H}$ on the $\mathrm{C}=\mathrm{O}$ of $\mathbf{1}$ with the formation of intermediate 13. Elimination of RNCS from $\mathbf{1 3}$ afforded the formation of $\mathbf{8}$ (Scheme 4). 
$2 \mathrm{CH}_{2} \mathrm{Cl}_{2}, \mathrm{CH}_{3} \mathrm{OH}$, ethyl acetate and tetrahydrofuran. However, the yields of 7a-e decreased and in some cases

3 such as ethyl acetate and tetrahydrofuran only traces of 7a-e were observed detectable by TLC. The excess

4 of one of the reaction partners, namely diphenylcyclopropenone $\mathbf{1}$ or thiosemicarbazides 6a-e, led to a

5 significant decrease of the yields.

6

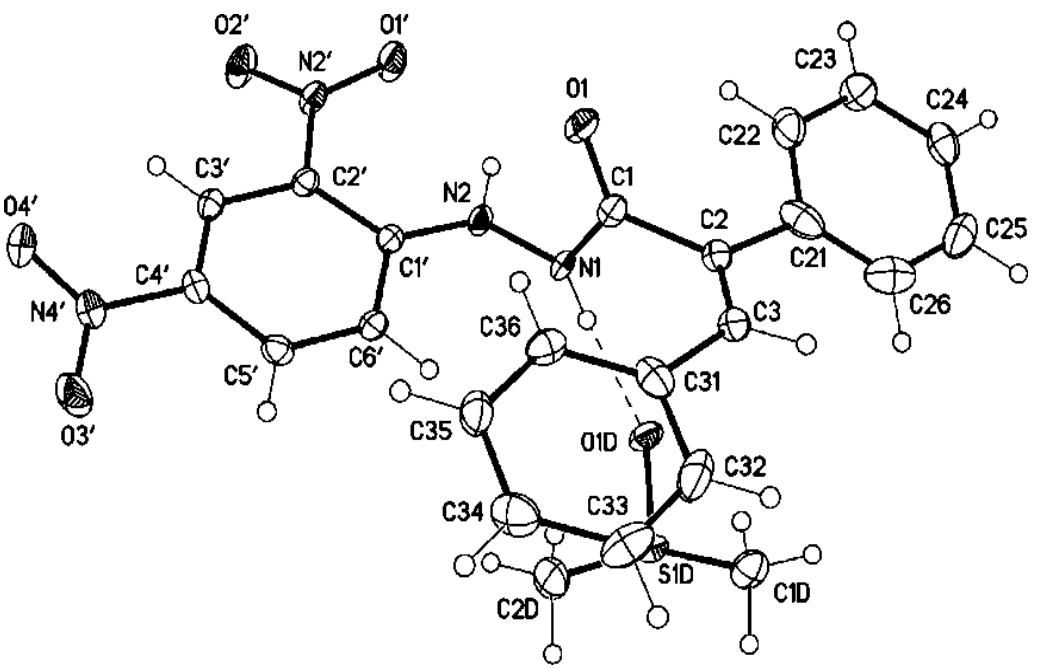

Figure 2: Molecular structure of $\mathbf{8}$ (minor disordered part and solvent omitted for clarity, displacement parameters are drawn at $50 \%$ probability level).<smiles>[R]NC(=S)Nc1ccc([N+](=O)[O-])cc1[N+](=O)[O-]</smiles>

6a-e
1<smiles>[R]NC(=S)Nc1ccc([N+](=O)[O-])cc1[N+](=O)[O-]</smiles>

13<smiles>CN(/C=C(/C(=O)NNc1ccc([N+](=O)[O-])cc1[N+](=O)[O-])c1ccccc1)S(=O)(=O)c1ccccc1</smiles>

8

9

Scheme 2: The plausible mechanism for the formation of $(Z)-N^{\prime}-(2,4-$ dinitrophenyl)-2,3diphenylacrylohydrazide $\mathbf{8}$. 


\section{Conclusion}

2 Nucleophilic attack of dinitrophenyl-4-substituted thiosemicarbazides on 2,3-diphenylcyclopropenone

3 afforded the formation of racemic 2-(2,4-dinitrophenyl)hydrazono)-5,6-diphenyl-1,3-thiazinan-4-ones as major products and (Z)-N'-(2,4-dinitrophenyl)-2,3-diphenylacrylohydrazide as minor product.

\section{Experimental}

7 Melting points were measured with Gallenkamp melting point apparatus. Infrared spectrum (IR) was 8 recorded with Alpha, Bruker FT-IR instruments taken as $\mathrm{KBr}$ discs. ${ }^{1} \mathrm{H}$ NMR at $400 \mathrm{MHz}$ and ${ }^{13} \mathrm{C}$ NMR at $100 \mathrm{MHz}$ on a Bruker AM 400 spectrometry with TMS as internal standard $(\delta=0)$, and data are reported as follows: Chemical shift, multiplicity $(\mathrm{s}=$ singlet, $\mathrm{d}=$ doublet, $\mathrm{t}=$ triplet, $\mathrm{q}=$ quartet, $\mathrm{m}=$ multiplet, $\mathrm{br}=$ broad). For ${ }^{13} \mathrm{C}$ NMR, TMS $(\delta=0)$ was used as internal standard and spectra were obtained with complete proton decoupling. Mass spectra were obtained using Finnigan MAT instrument ( $70 \mathrm{eV}$, EI-mode). Elemental analyses for $\mathrm{C}, \mathrm{H}, \mathrm{N}$, and $\mathrm{S}$ were carried out using an Elmyer 306. Preparative layer chromatography (plc) was carried out on glass plates covered with a $1.0 \mathrm{~mm}$ thick layer of slurry-applied silica gel $\left(\right.$ Merck $\left.\mathrm{Pf}_{254}\right)$.

\section{Starting materials}

The start materials 6a-e 2-(2,4-dinitrophenyl)- $N$-substituted hydrazinecarbothioamide were prepared from the reaction between 1-(3,5-dinitrophenyl) hydrazine and the corresponding isothiocyanates in absolute ethanol under refluxing temperature according to reported literatures [21,22]. 2,3-Diphenylcycloprop-2enone 1) was purchased from Fluka.

General procedure:

An equimolar amounts of 2,3-diphenylcycloprop-2-enone $\mathbf{1}$ and the appropriate 1,4-disubstituted thiosemicarbazides 6a-e were mixed in absolute ethanol and refluxed for about 4-6 h, furnished reddish orange precipitates of $\left(5 S^{*}, 6 S^{*}, Z\right)-2$-(2-(2,4-dinitrophenyl)hydrazono)-3-substituted-5,6-diphenyl-1,3thiazinan-4-one derivatives 7a-e and the residue were subjected to chromatographic separation using plc and toluene/ethyl acetate (10:3) as eluent to give (Z)- $N^{\prime}$-(2,4-dinitrophenyl)-2,3-diphenylacrylohydrazide 8 as a separated zone. 
Reddish orange crystals (acetonitrile), yield $470 \mathrm{mg}(81 \%), \mathrm{mp} .240-242{ }^{\circ} \mathrm{C}$; IR (KBr) v: 3265 (NH), 3072 (Ar-CH), $2928\left(\right.$ ali-CH), $1681(\mathrm{C}=\mathrm{O}), 1612(\mathrm{C}=\mathrm{N}), 1579(\mathrm{Ar}-\mathrm{C}=\mathrm{C}), 1530$ and $1333 \mathrm{~cm}^{-1}\left(\mathrm{NO}_{2}\right) ;{ }^{1} \mathrm{H} \mathrm{NMR}^{2}$ $\left(400 \mathrm{MHz}, \mathrm{CDCl}_{3}\right) \delta: 4.56-4.57(\mathrm{dd}, 1 \mathrm{H}, J=4.0 \mathrm{~Hz}$, thiazinanone-H6), 5.03-5.04 (dd, $1 \mathrm{H}, J=4.0 \mathrm{~Hz}$ thiazinanone-H5), 5.40-5.43 (d, 1H, $J=15.0 \mathrm{~Hz}, \mathrm{CH}_{2}$-benzyl), 5.61-5.64 (d, 1H, $J=15.0 \mathrm{~Hz}, \mathrm{CH}_{2}$-benzyl), 6.81-6.88 (m, 3H, Ar-H), 7.13-7.56 (m, 13H, Ar-H), 8.27-8.30 (m, 1H, Ar-H), 9.10 (m, 1H, Ar-H), 11.02 (br, $1 \mathrm{H}$, hydrazo-NH); ${ }^{13} \mathrm{C}$ NMR $\left(100 \mathrm{MHz}, \mathrm{CDCl}_{3}\right) \delta: 47.15$ (thiazinanone-CH-6), 47.90 (thiazinanone-CH5), $56.36\left(\mathrm{CH}_{2} \mathrm{Ph}\right), 116.05,123.51,127.47,128.01,128.16,128.32,128.44,128.67,128.76,129.43,130.08$, $130.25(\mathrm{Ar}-\mathrm{CH}), 129.21,132.51,133.77,137.05,137.87(\mathrm{Ar}-\mathrm{C}), 144.61(\mathrm{Ar}-C-\mathrm{NH}), 146.67(\mathrm{C}=\mathrm{N}), 168.85$ $(\mathrm{C}=\mathrm{O}) ; \mathrm{MS}(\mathrm{m} / \mathrm{z}): 553\left(\mathrm{M}^{+}, 47\right), 462$ (13), 347 (10), 207 (51), 182 (58), 149 (56), 91 (100), 77 (15); Anal. Calcd. for $\mathrm{C}_{29} \mathrm{H}_{23} \mathrm{~N}_{5} \mathrm{O}_{5} \mathrm{~S}$ (553.59): C, 62.92, H, 4.19, N, 12.65, S, 5.79. Found: C, 62.79, H, 4.06, N, 12.47, S, 5.65 .

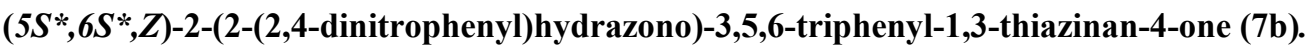

Reddish orange crystals (acetonitrile), yield $447 \mathrm{mg}$ (83\%), mp. 230-232 ${ }^{\circ} \mathrm{C}$; IR (KBr) v: $3262(\mathrm{NH}), 3076$ (Ar-CH), 2932-2922 (ali-CH), $1685(\mathrm{C}=\mathrm{O}), 1615(\mathrm{C}=\mathrm{N}), 1577(\mathrm{Ar}-\mathrm{C}=\mathrm{C}), 1532$ and $1330 \mathrm{~cm}^{-1}\left(\mathrm{NO}_{2}\right) ;{ }^{1} \mathrm{H}$ NMR (400 MHz, $\left.\mathrm{CDCl}_{3}\right) \delta:$ 4.54-4.55 (d, $1 \mathrm{H}, J=4.12 \mathrm{~Hz}$, thiazinanone-H6), 5.18-5.19 (d, $1 \mathrm{H}, J=4.12 \mathrm{~Hz}$, thiazinanone-H5), 6.80-7.00 (m, 4H, Ar-H), 7.10-7.32 (m, 9H, Ar-H), 7.35-7.52 (m, 3H, Ar-H), 8.04 (m, $1 \mathrm{H}$, Ar-H), 8.98 (m, 1H, Ar-H), 10.98 (br, hydrazo-NH); ${ }^{13} \mathrm{C}$ NMR (100 MHz, $\mathrm{CDCl}_{3}$ ) $\delta: 47.60$ (thiazinanoneCH5), 47.95 (thiazinanone-CH6), 115.97, 123.62, 127.71, 127.99, 128.39, 128.87, 129.35, 129.62, 130.08, 130.66, 131.03, 131.21 (Ar-CH), 129.35, 132.32, 133.77, 136.88, 137.86 (Ar-C), 144.57 (Ar-C-NH), 147.31 $(\mathrm{C}=\mathrm{N}), 168.70(\mathrm{C}=\mathrm{O})$; MS (m/z): $539\left(\mathrm{M}^{+}, 62\right), 462$ (23), 357 (48), 207 (61), 135 (37), 77 (100); Anal. Calcd. for $\mathrm{C}_{28} \mathrm{H}_{21} \mathrm{~N}_{5} \mathrm{O}_{5} \mathrm{~S}$ (539.56): C, 62.33; H, 3.92; N, 12.98; S, 5.94. Found: C, 62.18; H, 3.77; N, 12.87; S, 5.86 .

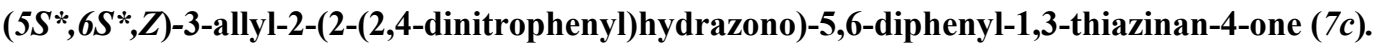

Reddish orange crystals (acetonitrile), yield $402 \mathrm{mg}(80 \%)$, mp. 208-210 ${ }^{\circ}$; IR (KBr) v: 3242 (NH), 3088 (Ar-CH), 2959-2925 (ali-CH), $1682(\mathrm{C}=\mathrm{O}), 1614(\mathrm{C}=\mathrm{N}), 1587(\mathrm{Ar}-\mathrm{C}=\mathrm{C}), 1529$ and $1342 \mathrm{~cm}^{-1}\left(\mathrm{NO}_{2}\right) ;{ }^{1} \mathrm{H}$ NMR (400 MHz, $\left.\mathrm{CDCl}_{3}\right) \delta: 4.42-4.43(\mathrm{~d}, 1 \mathrm{H}, J=4.08 \mathrm{~Hz}$, thiazinanone-H6), 4.70-4.92 (m, 2H, allyl$\left.\mathrm{CH}_{2} \mathrm{~N}\right), 4.96-4.97\left(\mathrm{~d}, 1 \mathrm{H}, J=4.08 \mathrm{~Hz}\right.$, thiazinanone-H5), 5.20-5.45 (m, 2H, allyl- $\left.\mathrm{CH}_{2}=\right), 5.90-6.10(\mathrm{~m}, 1 \mathrm{H}$, allyl-CH=), 6.60-6.70 (m, 2H, Ar-H), 6.75-6.82 (m, 2H, Ar-H), 7.00-7.30 (m, 6H, Ar-H), 7.65 (m, 1H, Ar$\mathrm{H}), 8.24(\mathrm{~m}, 1 \mathrm{H}, \mathrm{Ar}-\mathrm{H}), 9.05(\mathrm{~m}, 1 \mathrm{H}, \mathrm{Ar}-\mathrm{H}), 11.02\left(\mathrm{br}, 1 \mathrm{H}\right.$, hydrazo-NH); ${ }^{13} \mathrm{C}$ NMR $\left(100 \mathrm{MHz}, \mathrm{CDCl}_{3}\right) \delta$ : 
47.05 (allyl- $\mathrm{CH}_{2} \mathrm{~N}$ ), 47.15 (thiazinanone-CH6), 47.92 (thiazinanone-CH5), 118.87 (allyl- $\mathrm{CH}_{2}=$ ), 115.99, 123.60, 128.15, 128.31, 128.41, 129.24, 130.15, 130.22, 130.68 (Ar-CH), 133.75 (allyl-CH=), 128.79, 131.89, 133.69, $137.84\left(\right.$ Ar-C), $144.66($ Ar- $C-\mathrm{NH}), 146.32(\mathrm{C}=\mathrm{N}), 168.43(\mathrm{C}=\mathrm{O}) ; \mathrm{MS}(\mathrm{m} / \mathrm{z}): 503\left(\mathrm{M}^{+}, 72\right)$, 457 (18), 404 (26), 207 (55), 182 (100), 99 (66), 77 (87); Anal. Calcd. for $\mathrm{C}_{25} \mathrm{H}_{21} \mathrm{~N}_{5} \mathrm{O}_{5} \mathrm{~S}$ (503.53): C, 59.63; H, 4.20; N, 13.91; S, 6.37. Found: C, 59.45; H, 4.07; N, 13.74; S, 6.24.

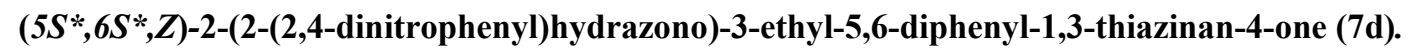

Reddish orange crystals (acetonitrile), yield $387 \mathrm{mg}$ (79\%), mp. 200-202 ${ }^{\circ}$; IR (KBr) v: 3238 (NH), 3093 (Ar-CH), 2935-2923 (ali-CH), $1678(\mathrm{C}=\mathrm{O}), 1616(\mathrm{C}=\mathrm{N}), 1585(\mathrm{Ar}-\mathrm{C}=\mathrm{C}), 1526$ and $1330 \mathrm{~cm}^{-1}\left(\mathrm{NO}_{2}\right) ;{ }^{1} \mathrm{H}$ NMR (400 MHz, $\left.\mathrm{CDCl}_{3}\right) \delta: 1.35\left(\mathrm{t}, 3 \mathrm{H}, J=7.77 \mathrm{~Hz}, \mathrm{CH}_{3}\right), 4.15-4.18\left(\mathrm{q}, 2 \mathrm{H}, J=7.77 \mathrm{~Hz}, \mathrm{CH}_{2}\right), 4.45-4.46$ (d, $1 \mathrm{H}, J=4.10 \mathrm{~Hz}$, thiazinanone-H6), 4.96-4.97 (d, 1H, $J=4.10 \mathrm{~Hz}$, thiazinanone-H5), 6.64-6.70 (m, 2H, Ar-H), 6.76-6.81 (m, 2H, Ar-H), 7.06-7.09 (m, 6H, Ar-H), 7.68 (m, 1H, Ar-H), 8.30 (m, 1H, Ar-H), 9.03 (m, 1H, Ar-H), 11.04 (br, 1H, hydrazo-NH); ${ }^{13} \mathrm{C}$ NMR (100 MHz, $\left.\mathrm{CDCl}_{3}\right) \delta: 12.86\left(\mathrm{CH}_{3}\right), 29.06\left(\mathrm{CH}_{2}\right), 47.20$, 47.70 (thiazinanone-CH6,5), 116.11, 123.63, 128.00, 128.30, 128.79, 129.22, 129.78, 130.10, 130.47 (Ar$\mathrm{CH}), 129.12,133.43,133.80,134.00,137.87(\mathrm{Ar}-\mathrm{C}), 144.62(\mathrm{Ar}-\mathrm{C}-\mathrm{NH}), 146.63(\mathrm{C}=\mathrm{N}), 168.60(\mathrm{C}=\mathrm{O})$; MS $(\mathrm{m} / \mathrm{z}): 491\left(\mathrm{M}^{+}, 48\right), 462(27), 445$ (35), 402 (19), 182 (100), 87 (70), 77 (91); Anal. Calcd. for $\mathrm{C}_{24} \mathrm{H}_{21} \mathrm{~N}_{5} \mathrm{O}_{5} \mathrm{~S}$ (491.52): C, 58.65; H, 4.31; N, 14.25; S, 6.52. Found: C, 58.51; H, 4.20; N, 14.07; S, 6.38.

\section{$\left(5 S^{*}, 6 S^{*}, Z\right)-3-c y c l o h e x y l-2-(2-(2,4-d i n i t r o p h e n y l) h y d r a z-o n o)-5,6-d i p h e n y l-1,3-t h i a z i n-a n-4-o n e ~(7 e)$.}

Reddish orange crystals (acetonitrile), yield $436 \mathrm{mg}$ (80\%), mp. 236-238 ${ }^{\circ} \mathrm{C}$; IR (KBr) v: 3229 (NH), 3090 (Ar-CH), 2938-2923 (ali-CH), $1675(\mathrm{C}=\mathrm{O}), 1613(\mathrm{C}=\mathrm{N}), 1586(\mathrm{Ar}-\mathrm{C}=\mathrm{C}), 1527$ and $1331 \mathrm{~cm}^{-1}\left(\mathrm{NO}_{2}\right) ;{ }^{1} \mathrm{H}$ NMR (400 MHz, $\left.\mathrm{CDCl}_{3}\right) \delta:$ 1.08-1.98 (m, 10H, cyclohexyl-CH$), ~ 2.02-2.46(\mathrm{~m}, 1 \mathrm{H}$, cyclohexyl-CH), 4.35$4.36(\mathrm{~d}, 1 \mathrm{H}, J=4.11 \mathrm{~Hz}$, thiazinanone-H6), 4.80-4.81 (d, 1H, $J=4.11 \mathrm{~Hz}$, thiazinanone-H5), 6.62-6.70 (m, 2H, Ar-H), 6.84-6.92 (m, 2H, Ar-H), 7.04-7.46 (m, 6H, Ar-H), 7.71 (m, 1H, Ar-H), 8.32 (m, 1H, Ar-H), 9.10 (m, 1H, Ar-H), 11.00 (br, hydrazo-NH); $\left.{ }^{13} \mathrm{C} \mathrm{NMR} \mathrm{(100} \mathrm{MHz,} \mathrm{CDCl}_{3}\right) \delta: 25.74,26.53,29.78$ (cyclohexyl$\mathrm{CH}_{2}$ ), 47.63, 47.91 (thiazinanone-CH6,5), 57.08 (cyclohexyl-CH), 115.93, 123.64, 128.04, 128.22, 128.71, 129.57, 130.25, 130.37, 130.77 (Ar-CH), 129.11, 133.38, 133.69, 137.96 (Ar-C), 144.59 (Ar-C-NH), 146.51 $(\mathrm{C}=\mathrm{N}), 168.87(\mathrm{C}=\mathrm{O})$; MS (m/z): $545\left(\mathrm{M}^{+}, 51\right), 462$ (43), 499 (28), 455 (39), 182 (100), 141 (68), 77 (91); Anal. Calcd. For $\mathrm{C}_{28} \mathrm{H}_{27} \mathrm{~N}_{5} \mathrm{O}_{5} \mathrm{~S}$ (545.61): C, 61.64; H, 4.99; N, 12.84; S, 5.88. Found: C, 61.48; H, 4.83; N, 12.74; S, 5.75 .

(Z)-N'-(2,4-dinitrophenyl)-2,3-diphenylacrylohydrazide (8). 
1 Yellow crystals (acetonitrile), yield 8-12\%, mp. 168-169 C; IR (KBr) v: 3247 (NH), 3130 (Ar-H), 2930 (ali-

$2 \mathrm{H}), 1693(\mathrm{C}=\mathrm{O}), 1591(\mathrm{Ar}-\mathrm{C}=\mathrm{C}), 1542$ and $1334 \mathrm{~cm}^{-1}\left(\mathrm{NO}_{2}\right) ;{ }^{1} \mathrm{H}$ NMR $\left(400 \mathrm{MHz}, \mathrm{CDCl}_{3}\right) \delta: 6.42(\mathrm{~s}, 1 \mathrm{H}$,

3 acryl-CH), 7.10-7.55 (m, 10H, Ar-H), 7.94 (m, 1H, Ar-H), 8.48 (br, 1H, NH), 9.04 (m, 1H, Ar-H), 9.43 (br,

$41 \mathrm{H}$, amide-NH); ${ }^{13} \mathrm{C}$ NMR $\left(100 \mathrm{MHz}, \mathrm{CDCl}_{3}\right) \delta: 117.65,123.18,126.17,127.52,128.15,128.26,129.19$,

5 129.38, 129.85 (Ar-CH), 133.68, 139.75 (C1 and C2-acrylohydrazide); 130.00, 134.34, 135.60, 137.80 (Ar-

$6 \mathrm{C}) ; 145.10(\mathrm{Ar}-\mathrm{C}-\mathrm{NH}), 165.18(\mathrm{C}=\mathrm{O}) ; \mathrm{MS}(\mathrm{m} / \mathrm{z}): 404\left(\mathrm{M}^{+}, 100\right), 356(23), 221(26), 205(13), 195(18), 181$

7 (32), 138 (20), 77 (41); Anal. Calcd. for $\mathrm{C}_{21} \mathrm{H}_{16} \mathrm{~N}_{4} \mathrm{O}_{5}$ (404.38): C, 62.37; H, 3.99; N, 13.86. Found: C, 62.25;

$8 \quad \mathrm{H}, 3.86 ; \mathrm{N}, 13.77$.

\section{Single crystal X-ray structure determination of 7a and 8:}

Suitable crystals were obtained by recrystallization from acetonitrile. The single-crystal X-ray diffraction study were carried out on a Bruker D8 Venture diffractometer with Photon100 detector at 123(2) K using $\mathrm{Cu}-\mathrm{K} \alpha$ radiation $(\lambda=1.54178 \AA)$. Direct Methods for 7a (SHELXS-97) [23] and dual space methods for 8 (SHELXT) [24] were used for structure solution and refinement was carried out using SHELXL-2014 (fullmatrix least-squares on $F^{2}$ ) [25]. Hydrogen atoms were localized by difference electron density determination and refined using a riding model $(\mathrm{H}(\mathrm{N})$ free $)$. Semi-empirical absorption corrections were applied. For 8 an extinction correction was applied. In 8 the 2,3-diphenylacrylo substituent is disordered (see cif-files for details).

Compound 7a: red crystals, $\mathrm{C}_{29} \mathrm{H}_{23} \mathrm{~N}_{5} \mathrm{O}_{5} \mathrm{~S}, \mathrm{Mr}=553.58$, crystal size $0.24 \times 0.06 \times 0.02 \mathrm{~mm}$, monoclinic, space group $P 2{ }_{1} / c$ (No. 14), $a=19.9611(6) \AA, b=11.4148(4) \AA, c=10.9667(4) \AA, \beta=93.844(1)^{\circ}, V=$ $2493.16(15) \AA^{3}, Z=4, \rho=1.475 \mathrm{Mg} / \mathrm{m}^{-3}, \mu(\mathrm{Cu}-\mathrm{K} \alpha)=1.601 \mathrm{~mm}^{-1}, F(000)=1152,2 \square_{\max }=144.2^{\circ}, 21195$ reflections, of which 4907 were independent $\left(R_{\text {int }}=0.030\right), 364$ parameters, 1 restraint, $R_{1}=0.046$ (for 4478 $I>2 \sigma(I)), w R_{2}=0.118$ (all data), $S=1.08$, largest diff. peak / hole $=0.723 /-0.490$ e $\AA^{-3}$.

Compound 8: yellow crystals, $\mathrm{C}_{21} \mathrm{H}_{16} \mathrm{~N}_{4} \mathrm{O}_{5} \cdot \mathrm{C}_{2} \mathrm{H}_{6} \mathrm{OS}, M r=482.50$, crystal size $0.32 \times 0.16 \times 0.12 \mathrm{~mm}$, triclinic, space group $P-1$ (No. 2), $a=9.0191(3) \AA, b=11.3050(3) \AA, c=11.8112(3) \AA, \alpha=87.986(1)^{\circ}, \beta=$ $69.079(1)^{\circ}, \gamma=79.272(1)^{\circ}, V=1104.58(6) \AA^{3}, Z=2, \rho=1.451 \mathrm{Mg} / \mathrm{m}^{-3}, \mu(\mathrm{Cu}-\mathrm{K} \alpha)=1.732 \mathrm{~mm}^{-1}, F(000)=$ $504,2 \theta_{\max }=144.0^{\circ}, 15897$ reflections, of which 4309 were independent $\left(R_{\text {int }}=0.022\right), 313$ parameters, 10 restraints, $R_{l}=0.038$ (for $\left.4234 I>2 \sigma(I)\right), w R_{2}=0.093$ (all data), $S=1.06$, largest diff. peak $/$ hole $=0.584 /$ 0.369 e $\AA^{-3}$. 


\section{Supporting Information}

2 CCDC 1840977 (7a), and 1840978 (8) contain the supplementary crystallographic data for this paper. These

3 data can be obtained free of charge from The Cambridge Crystallographic Data Centre via www.ccdc.cam.ac.uk/data request/cif

5

\section{Acknowledgment}

7 Alaa A. Hassan is indebted to the AvH-Foundation for the donation of a Shimadzu 408 IR instrument.

\section{$9 \quad$ References}

1. Rawal RK, Tripathi R, Katti SB, Pannecouque C, De Clercq E (2007) Synthesis and evaluation of 2-(2,6-dihalophenyl)-3-pyrimidinyl-1,3-thiazolidin-4-one analogues as anti-HIV-1 agents. Bioorg. Med. Chem. 15: 3134-3142. doi:10.1016/j.bmc.2007.02.044

2. Rawal RK, Tripathi R, Katti SB, Pannecouque C, De Clercq E (2007) Design, synthesis, and evaluation of 2-aryl-3-heteroaryl-1,3-thiazolidin-4-ones as anti-HIV agents. Bioorg. Med. Chem. 15: 1725-1731. doi:10.1016/j.bmc.2006.12.003

3. Verma A, Saraf SK (2008) 4-Thiazolidinone-A biologically active scaffold. Eur. J. Med. Chem. 43: 897-905. doi:10.1016/j.ejmech.2007.07.017

4. Kamel MM, Ali HI, Anwar MM, Mohamed NA, Soliman AM (2010) Synthesis, antitumor activity and molecular docking study of novel Sulfonamide-Schiff's bases, thiazolidinones, benzothiazinones and their C-nucleoside derivatives. Eur. J. Med. Chem. 45: 572-580. doi:10.1016/j.ejmech.2009.10.044

5. Verma A, Verma SS, Saraf SK (2010) A DIC mediated expeditious small library synthesis and biological activity of thiazolidin-4-one and 1,3-thiazinan-4-one derivatives. J. Heterocycl. Chem. 47: 1084-1089. DOI 10.1002/jhet.429

6. Kumawat MK, Singh UP, Singh B, Prakash A, Chetia D (2016) Synthesis and antimalarial activity evaluation of 3-(3-(7-chloroquinolin-4-ylamino)propyl)-1,3-thiazinan-4-one derivatives. Arabian J. Chem. 9: S643-S647. http://dx.doi.org/10.1016/j.arabjc.2011.07.007 
7. Bosenbecker J, Bareño VDO, Difabio R, Vasconcellos FA, Dutra FSP, Oliveira PS, Barschak AG, Stefanello FM, Cunico WJ (2014) Synthesis and Antioxidant Activity of 3-(Pyridin-2-ylmethyl)1,3-thiazinan(thiazolidin)-4-ones. Biochem. Molecular Toxicology 28: 425-432. DOI $10.1002 / \mathrm{jbt} .21581$

8. Zebardast T, Zarghi A, Daraie B, Hédayati M, Dadrass OG (2009) Design and synthesis of 3-alkyl2-aryl-1,3-thiazinan-4-one derivatives as selective cyclooxygenase (COX-2) inhibitors. Bioorg. Med. Chem. Lett. 19: 3162-3165. doi:10.1016/j.bmcl.2009.04.125

9. Eicher T, Franke G (1981) On the Reaction of Diphenylcyclopropenone with Guanidines. Liebigs Ann. Chem. 1337-1353. https://doi.org/10.1002/jlac.198119810802

10. Takahashi M, Funaki T, Honda H, Yokoyama Y, Takimoto H (1982) Formation of 1,2,3,5Tetrasubstituted 2-Pyrrolin-4-ones and 1,2,3-Trisubstituted Pyrroles from Diphenylcyclopropenone and 1,4-Diazabutadienes. Heterocycles 19: 1921-1924. DOI: 10.3987/R-1982-10-1921

11. Gomaa MAM, Döpp D (2003) Reaction of $N^{1}, N^{2}$-Diarylamidines with (2,3-Diphenylcyclopropen1-ylidene)propanedinitrile: Synthesis of [2-Arylamino-4(1H)-pyridinylidene-[propanedinitriles. Synthesis 10: 1545-1548. DOI: 10.1055/s-2003-40522

12. Gomaa MAM (2002) Reaction of diimines and azines with diphenylcyclopropenone. J. Chem. Soc.: Perkin Trans. 1: 341-344. DOI: 10.1039/b109711n

13. Hassan AA, Abdel-Latif FF, Nour El-Din AM, Mostafa SM, Nieger M, Bräse S (2012) Synthesis of (E)-2,5-disubstituted 1,3,4-thiadiazolyl-2,3-diphenylpropenones from alkenylidenehydrazinecarbothioamides. Tetrahedron 68: 8487-8492. http://dx.doi.org/10.1016/j.tet.2012.07.063

14. Aly AA, Hassan AA, Ameen MA, Brown AB (2008) Chemistry of cyclopropenones: synthesis of new pyrrolo[2,1-b]-1,3,4-oxadiazoles. Tetrahedron Lett. 49: 4060-4062. doi:10.1016/j.tetlet.2008.04.066

15. Aly AA, Hassan AA, Gomaa MAM, El-Sheref EM (2007) Unusual reactivity of thiosemicarbazides towards-2,3-diphenylcyclopropenone: synthesis of new pyridazinethiones and 1,2,4-triazolo[4,3b]pyridazinethiones. Arkivoc (xiv): 1-11. DOI: http://dx.doi.org/10.3998/ark.5550190.0008.e01

16. Clayden J, MacLellan P (2011) Asymmetric synthesis of tertiary thiols and thioethers. Beilstein J. Org. Chem. 7: 582-595. doi:10.3762/bjoc.7.68 
17. Damani LA (1989) "Sulphur-Containing Drugs and Related Organic Compounds" Ed. Wiley: NewYork.

18. Niu B, Jiang B, Yua LZ, Shi M (2018) Base-promoted [3 + 3] cyclization of cyclopropenones and cyclopropenethiones with amides for the synthesis of 6H-1,3-oxazin-6-ones and 6H-1,3-thiazin-6ones. Org, Chem. Front. 5: 1267-1271. DOI: 10.1039/c8qo00091c

19. Gomaa, MAM (2004) Reaction of $N^{1}, N^{2}$-Diarylamidines with 2,3-Diphenylcyclopropenone. Z. Naturforsch, 59b: 597-600. http://znaturforsch.com

20. Shan L, Wu G, Liu M, Gao W, Ding J, Huang X, Wu H (2018) $\alpha, \beta$-Diaryl unsaturated ketones via palladiumcatalyzed ring-opening of cyclopropenones with organoboronic acids. Org, Chem. Front. 5: 1651-1654. DOI: $10.1039 / \mathrm{c} 8 \mathrm{qo} 00241 \mathrm{j}$

21. Parashar B, Jain A, Bharadwaj S, Sharma VK (2012) Synthesis and pharmacological properties of some novel pyrazolidine and pyrazole derivatives. Med. Chem. Res. 21:1692-1699. DOI 10.1007/s00044-011-9687-0

22. Begum S, Choudhary MI, Khan KM (2009) Synthesis, phytotoxic, cytotoxic, acetylcholinesterase and butrylcholinesterase activities of $N, N^{\prime}$-diaryl unsymmetrically substituted thioureas. Natural Product Research 23:1719-1730. DOI: 10.1080/14786410802223778

23. Sheldrick GM (2008) A short history of SHELX. Acta Cryst. A64: 112-122. doi:10.1107/S0108767307043930

24. Sheldrick GM (2015) SHELXT-Integrated space-group and crystal-structure determination. Acta Cryst. A71: 3-8. doi:10.1107/S2053273314026370

25. Sheldrick GM (2015) Crystal structure refinement with SHELXL. Acta Cryst. C71: 3-8. doi:10.1107/S2053229614024218 